\title{
The effect of a gold coin fine on C-reactive protein test ordering in a tertiary referral emergency department
}

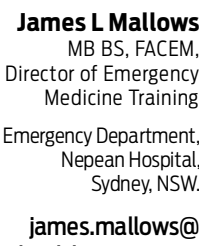

health.nsw.gov.au

MJA 2013; 199: 813-814 doi: 10.5694/mjal3.11298

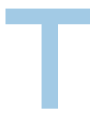

esting for C-reactive protein (CRP), an acute-phase reactant, is used in investigations for patients who present to emergency departments (EDs). It has been assessed for such roles as identifying bacteraemia in febrile patients, ${ }^{1-4}$ evaluating patients with an acute abdomen, 5,6 and diagnosing patients with possible osteomyelitis or septic arthritis. ${ }^{7}$ However, such studies have not shown the CRP test to be useful for any of these indications, or indeed for most conditions for which patients present to EDs. Despite this paucity of evidence, CRP tests continue to be ordered as part of the work-up for patients presenting to EDs.

Nepean Hospital is a tertiary referral hospital that serves the western suburbs of Sydney in New South Wales, Australia. About 60000 patients attend its ED each year. The ED has a $20 \%$ paediatric caseload and a $36 \%$ admission rate.

This study resulted from an audit of pathology test ordering in the Nepean Hospital ED. The aim of the audit was to determine what and how many tests were being ordered, the cost to the department and who was being billed for the tests. Also examined was how pathology testing was affecting our National Emergency Access Target (NEAT) times. To our horror, we discovered that about $25 \%$ of patients presenting to the ED had a CRP test as part of their work-up. This included patients who presented with problems that were likely to be non-infective, such as chest pain. A rough backof-the-napkin calculation suggested that we could afford a new bedside ultrasound machine every year with the money spent on CRP tests ordered in the ED. Also, we found that it took up to 2 hours to get the CRP test result, putting our NEAT performance at risk. Furthermore, we noted that there is evidence that

Abstract

Objective: To examine the effect of an education campaign based around a gold coin fine on ordering of C-reactive protein (CRP) tests.

Design and setting: A retrospective analysis of CRP test ordering before and after the intervention in the emergency department (ED) of a tertiary referral hospital in metropolitan Sydney that sees about 60000 patients per annum. The date of the intervention - 2 August 2013 - corresponded with Jeans for Genes Day.

Main outcome measures: Number of CRP tests ordered in the ED.

Results: 1290 CRP tests were ordered before the intervention (1-31 July), and 394 were ordered after the intervention (2-31 August). This decrease in CRP test ordering was despite an increased number of ED presentations in August compared with July ( $5219 \vee 5497$ presentations). This represented an absolute reduction in the rate of CRP test ordering of $17.6 \%(95 \% \mathrm{Cl}, 16.2 \%-18.9 \%$; $P<0.001$ ).

Conclusion: The threat of a gold coin fine for ordering a CRP test, as part of a broader education campaign, significantly reduced the number of CRP tests ordered in a tertiary referral ED.

interventions aimed at reducing CRP test ordering are effective in an ED setting. ${ }^{8}$ We felt it was time to act, to reduce ordering of CRP tests in the Nepean Hospital ED.

The objective of this study was to assess the effect of an education campaign centring on a gold coin fine for CRP test ordering in the ED.

\section{Methods}

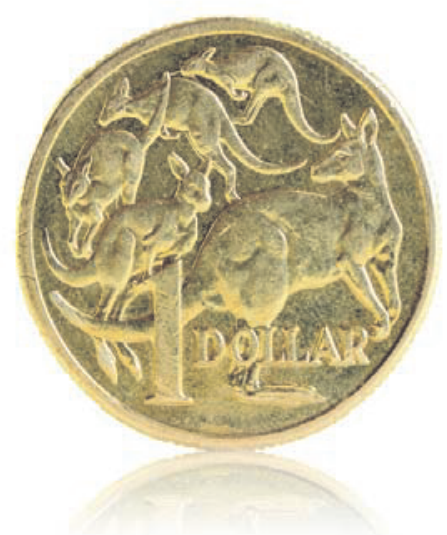

An education campaign on CRP testing was conducted at the Nepean Hospital ED to coincide with Jeans for
Genes Day on 2 August 2013. This day was characterised by an informal atmosphere, including much wearing of jeans, various morning and afternoon teas, and gold coin donations. "No CRP Day" was run simultaneously with Jeans for Genes Day at the Nepean Hospital ED. Education was commenced running up to the day, with email discussions about the utility and potential indications of CRP testing. Also discussed was the cost of CRP testing to the ED, what we could spend the money on otherwise, and how CRP tests were affecting our NEAT performance.

A CRP jar was used, akin to a swear jar in that anyone ordering a CRP test had to put a gold coin in the jar as penance. This money was slated to be donated to the Children's Medical Research Institute as part of Jeans for Genes Day. Education continued, centring on the CRP jar, with the jar being shaken loudly in front of anyone ordering a CRP test. The jar was left in the ED for the remainder of the month as visual reminder of the campaign.

A retrospective audit of CRP ordering was performed. The date of the 
intervention was defined as 2 August 2013, the control period was 1-31 July 2013 and the study period was 2-31 August 2013. The number of CRP tests ordered by medical staff working in the ED was collected for each period. A comparison of independent proportions was performed using VassarStats (http:// vassarstats.net).

\section{Results}

There were 5219 presentations in the control period and 5497 in the study period. In the control period, 1290 CRP tests were ordered, that is in $24.7 \%$ of presentations. In the study period, 394 CRPs tests were ordered, that is in $7.2 \%$ of presentations. This represented an absolute reduction in the rate of CRP test ordering of $17.6 \%$ (95\% CI, $16.2 \%-18.9 \% ; P<0.001)$.

\section{Discussion}

After No CRP Day, there was a significant reduction in the number of CRP tests ordered in the Nepean Hospital ED, beyond what was expected when the study was designed. It is possible that medical staff in the ED knew that CRP testing is not useful in the workup for most patients and that they needed "permission" not to order CRP tests. Once they had this permission, they were very happy not to order CRP tests, thus producing the massive fall in ordering.

A significant finding of this study was the proportion of presentations for which CRP tests were performed as part of the clinical work-up before the intervention: almost one-quarter of ED presentations. It is possible that there had been little thought behind pathology test ordering during the control period, akin to a scattergun approach. This is despite many clinical pathways being available for use in the ED, none of them advising the use of CRP testing.

Post-hoc discussions with medical students suggested that, in medical school, the CRP test was not taught as being an important part of a clinical work-up. Similar post-hoc discussions with senior, post-fellowship medical staff suggested that the CRP

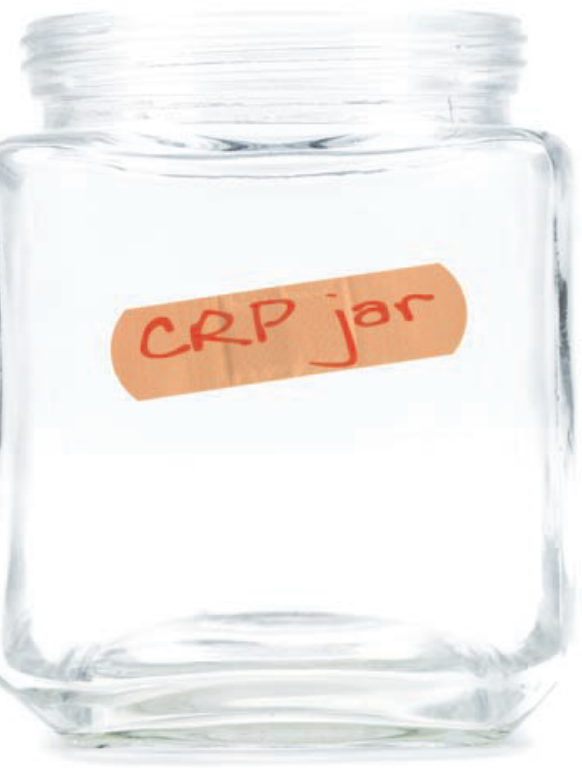

test was not widely regarded as an important test, with a few exceptions relative to craft group. It seemed then that the main group "championing" the use of CRP testing was the registrar and trainee cohort of medical staff. Medical graduates, as interns, became exposed to the use of the CRP test as a standard test for the work-up of patients in their medical practice, saw CRP testing as an entrenched part of practice, and transitioned into registrars teaching the concept of CRP testing as standard practice to future cohorts of medical graduates. Then it seems that once registrars completed their training and became specialists, the need for CRP testing became less important. Thus, like a virus requiring an endemic host population, it seems that the idea of CRP testing as an important part of a standard work-up is endemic to the hospital registrar population.

A limitation of this study is that only numbers of CRP tests ordered were examined, not the effects on patient outcomes or length of stay. Also, the study did not assess whether CRP testing was indicated in each patient for whom it was ordered, so it is not possi- ble to comment on the appropriateness of individual CRP tests. Hence there is no reason to advocate that CRP tests should never be ordered for patients who present to an ED.

The number of CRP tests ordered after the intervention seemed to still be high compared with the number of CRP tests claimed to be ordered by ED medical staff (zero!). This might be because CRPs were "added on", by inpatient teams, to pathology tests ordered by ED staff while patients were still in the ED or after patients were transferred to a ward. There was no way to control for this phenomenon in the study.

Very little money was generated for Jeans for Genes Day beyond what was raised by nursing staff at morning and afternoon teas, and a lot less than the expected \$394. This was probably more related to the propriety of the test orderers rather than a failure of the concept of the CRP jar.

Acknowledgements: I wish to thank Rod Bishop for input into the design of the study and contribution to the reference list, and Karla Barnes for collecting the data from the pathology department.

Competing interests: No relevant disclosures.

Received 10 Oct 2013, accepted 5 Nov 2013.

1 Adams N. Diagnostic use of C-reactive protein in bacteraemic emergency department patients. Emerg Med Australas 2005; 17: 371-375.

2 Wyllie DH, Bowler IC, Peto TE. Bacteraemia prediction in emergency medical admissions: role of C reactive protein. J Clin Pathol 2005; 58 : 352-356.

3 Ruiz-Esteban R, Sarabia PR, Delgado EG, et al. Procalcitonin and C-reactive protein levels as diagnostic tools in febrile patients admitted to a general internal medicine ward. Clin Biochem 2012; 45: 22-25.

4 Au-Yong A. Towards evidence-based emergency medicine: best BETs from the Manchester Royal Infirmary. BET 2: C-reactive protein in the diagnosis of bacteraemia. Emerg Med J 2012; 29: 423-424.

5 Salem TA, Molloy RG, O'Dwyer PJ. Prospective study on the role of C-reactive protein (CRP) in patients with an acute abdomen. Ann R Coll Surg Engl 2007; 89: 233-237.

6 Coyle JP, Brennan CR, Parfrey SF, et al. Is serum Creactive protein a reliable predictor of abdominopelvic CT findings in the clinical setting of the non-traumatic acute abdomen? Emerg Radiol 2012; 19: 455-462.

7 Margaretten ME, Kohlwes J, Moore D, Bent S. Does this patient have septic arthritis? JAMA 2007; 297: 1478-1488.

8 Santos IS, Bensenor IM, Machado JB, et al. Intervention to reduce C-reactive protein determination requests for acute infections at an emergency department. Emerg Med J 2012; 29: 965-968. 